\title{
Readers' Forum-13
}

\author{
Compiled by \\ Vinod H. Shah \\ Shruti' ENT Hospital \\ Opp.Sub Jail Ring Road \\ Fax : 91-261-665757
}

\section{Q. 1 What is the current status of intact canal wall tympanoplasty and in which cases of CSOM you will prefer to do the same?}

A. The current status of Intact Canal Wall Tympanoplasty: (ICWT) phrase should be defined-it should mean mastoid surgery where conventionally you would not have taken down'the posterior canal wall. Classical Cortical Mastoidectomy should not be called ICWT.

The initial enthusiasm about ICWT has vained in cases of cholesteatomas as there is possibility of :

1. Residual Cholesteatoma

2. Recurrent Cholesteatoma

3. Iterogenic Cholesteatoma

I would do-in a case of Cholesteatoma-a canal wall downtechnique, or $A B M C$ (Paparella) or canal wall reconstruction after taking down the canal wall. The ICWT still has place-in granulating mastoiditis', a congenital cyst of mastoid and tensa retraction cholesteatoma where there is no involvement of aditus or antrum.

In children with cholesteatoma, ICWT would appear to be an advantage as post op dressings and cavity care are avoided, However regular follow up and timely revisions are essential. This is possible in advanced health care systems like scandinavion countries. Indeed it is not realistic in our country.

Dr. Sunil Mody

Rajkot

The current status of intact canal wall technique varies from surgeon to surgeon. I personally perform the technique for-

a) Surgeries on ears with safe disease i. e. mucosal / adhesive pathology.

b) Patients having limited attic cholesteatoma i.e. up to the aditus which has been removed in toto.

In only one patient have I had to perform an open technique even though he clinically had a safe disease. The patient presented with acute suppurative labyrinthitis, a small central perforation on examination and total S.N. loss. On exploration patient had externsive granulations in the mastoid and a fistula on the lateral semicircular canal.

Dr. Neelam Vaid Pune

In my practice; I reserve the intact canal wall tympanoplasty for safe otitis media only and never for cholesteatoma. Rarely for "Granular Otitis", when I am sure about exenteration of all mastoid cells, I like to keep the canal wall intact. However, in paediatric cases, for safe disease, I invariably try and preserve the canal wall.

One modification of I.C.W. Tympanoplastywhere $I$ can not see the facial recess and sinus tympani I prefer to thin out the deep canal wall 
anteriorly \& tackle the disease anteriorly and at the same time, posteriorly to approach the rest fo the mastiod air cells.

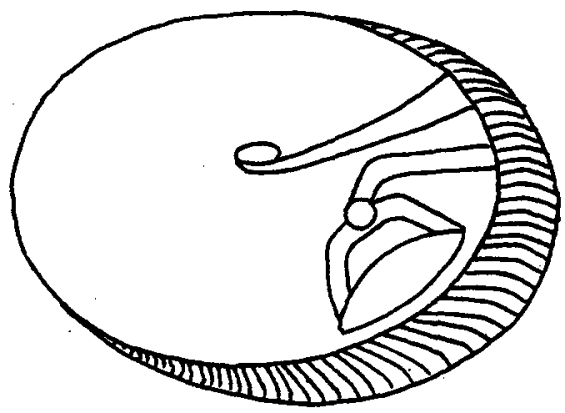

Widening of Bony Wall

This modified intact canal wall technique, I call it A-P technique (ant-post technique) where a limited disease like granulations even in anterior attic can be tackled by widening the anterior butteress anteriorly. At the end of surgery, this defect in the deep canal wall can be reinforced by a piece of tragal cartilage covered with perichondrium so as to reduce the extra widened ear canal to its original size. The attached perichondrium can well be used to create and reinforce the new drum.

\section{Dr. Kailash Sant Pune}

There is no doubt that world wide, the role of intact canal wall Tympanoplasty in cases of chronic suppurative otitis media has undergone drastic reduction. The initial fascination with the procedure in the $70^{\prime} \mathrm{s}$ and $80^{\prime} \mathrm{s}$ gave way to frustration, when many of the patients came . back with "Recurrent" or "Residual" cholesteatoma, However this should not undermine the basic elegance of the procedure.

I still do it and teach the procedure to my juniors. I particularly like to do the surgery in non-cholesteatomatous chronic otitis media with obstruction to the aditus and inadequate ventilation of the mastoid air cell system. This procedure is particularly useful when doing ossicular reconstruction as the posterior tympanotomy "window" affords an unparallelled view of the ossicular assembly, particularly with the stapes or the footplate. However in my view, there is no role for this operation in cholesteatoma. Again with the advent of cochlear implant surgery the intact canal wall technique is having resurgence.

Dr. Mohan Kameswaran Madras

I would prefer to do the same in the following cases of CSOM :

1. All cases of tubotympanic type of CSOM

2. Disease restricted to middle ear, attic, aditus and posterosuperior region which can be / and is completely removed and if.

3. The patient can come for regular follow-up examination.

\section{Dr. Gauri Mankekar Mumbai}

The concept of conservative surgery. Intact Canal Wall Tympanoplasty is preferred by most of the today's otologists. The reason behind this concept and the advantages of ICWT :

1. Establishes.good hearing mechanism.

2. Safe, easy and faster procedure.

3. No cavity problem.

I will prefer this procedure in case of :

1. Tubotympanic type of CSOM

2. Mucosal (granulation) disease

3. Chronic mastoiditis.

4. Retraction pocket. (Without cholesteatoma)

5. Facial nerve decompression.

\section{Dr Dipali Solanky Surat}

Intact Canal Wall Tympanoplasty or combined approach tympanoplasty for cholesteatoma avoids cavity" problems and is still a popular procedure in some western centres where they have a very good follow-up and second look operation is well accepted by patients. 
I prefer to do a canal down mastoidectomy with tympanoplasty procedure for most cases of cholesteatoma. However, for small retraction pockets with or without a cholesteatoma, I would do an intact canal procedure with reconstruction of the outer attic wall (using tragal cartilage) with tympanoplasty.

\section{Dr. Samir Bhargave Mumbai}

In the set up up of municipal hospital where patients come from various parts of country the overall follow up is poor. Patients do not agree for second look operation and rarely come back even if staged procedure is done. Many times patients have bilateral disease. The pre requisite for intact canal wall tympanoplasty i.e. second look operation can not be met with. Hence we prefer to do canal wall down procedure for cholesteatoma cases. Intact canal wall is done only of there is a small retraction pocket which is satisfactorily cleared and then attic defect is reconstructed using conchal cartilage.

Intact canal wall tympanoplasty is very good for educated patients who understand the necessity for second look operation as the cavity problems are eliminated

\section{Dr. Hetal Patel Mumbai}

The current status of intact canal wall tympanoplaty is one of the raging contraversies to date, all over the world. I would say that definite indications for intact canal wall procedures include all granulating otitis medias including tuberculos otitis media. In limited sinus tympani cholesteatoma and in epitympanic. cholesteatoma which does not extend beyond the horizontal part of the facial nerve and in those cases where the mastoid is well pneumatised with mid fossa dura and sinus plate allowing adequate exposure for an intact canal wall tympanoplasty.

As far as hearing results go, good reconstruction is possible in both intact canal and other procedures. In most centres I have visited in the west, they almost always do an intact canal wall procedure for cholesteatoma.. However, they can afford the luxury of second and third looks which is not possible in third world countries like ours, where patient follow-up is very unreliable. Hence 1 individualise each case, based on the nature and extent of the pathology, and the literacy / reliability of the patient, to make my decision.

\section{Dr. Aruna Visvanathan Coimabatore}

\section{Q. 2 What kind of prosthesis you will prefer to replace incus in ossiculoplasty? Kindly support your choice with your results.}

A. A plothora of prostheses are availableindicating that there is no satisfactory solution to the problem.

Bioactive, and bioinert prosthesis - all have interface with body's own living tissue and non living tissue. This results in erosion. Eustachian Tube problem, and infection and foreign body reaction often result in extrusin of foreign material.

So far living tissues are the best materials - bone or cartilage.

In order of preference :

1. patient's own incus - if healthy can be reshaped and malleus - incus - stapes assembly is made.

2. Homo graft incus - when available. This is generally possible in teaching instituies only.

3. Cortical bone - shape to fit and fill the gap between malleus and incus - with possibility of resorption.

4. Cartilage - Tragal and Conchal cartilage or even preserved septal cartilage are the next best materials. However, delayd softening and resorption are the delayed problems.

\section{Dr. Sunil Mody \\ Rajkot}

In ossiculoplasty I replace incus with tragal cartilage in cases of cholesteatoma and autologous bone in non cholesteatoma cases. If bone is insufficient I would use cartilage. I have not used any other material except gold prosthesis in 2 cases. Both the cases had no extrusion and good postoperative hearing improvement.

Dr Neelam Vaid Pune 
The autograft cartilage (tragal) is my choice of prosthesis to replace incus. The tragal cartilage piece with attached perichondrium is used either single piece ( if depth of middle ear is good) or two pieces ( if depth of middle ear is less and / or the stapes is very horizontal in position)

Perichondrial Graft

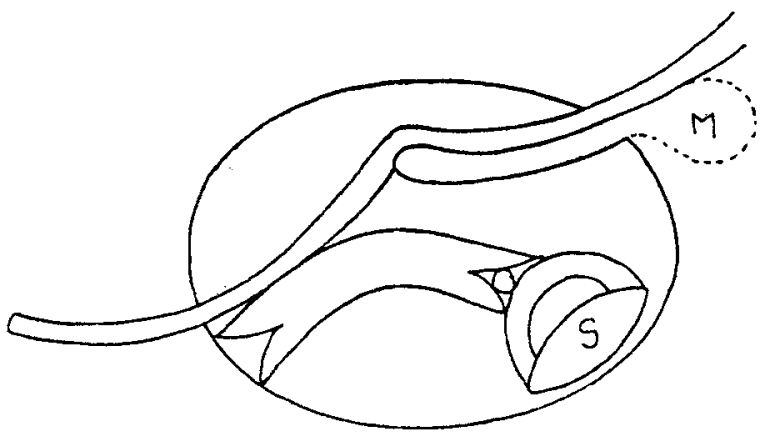

Single

Piece Cartilage

Single piece tragal cartilage with perichondrium I R Prosthesis, by passing the malleus. I have nearly stopped using metal ( Gold) for IRP.

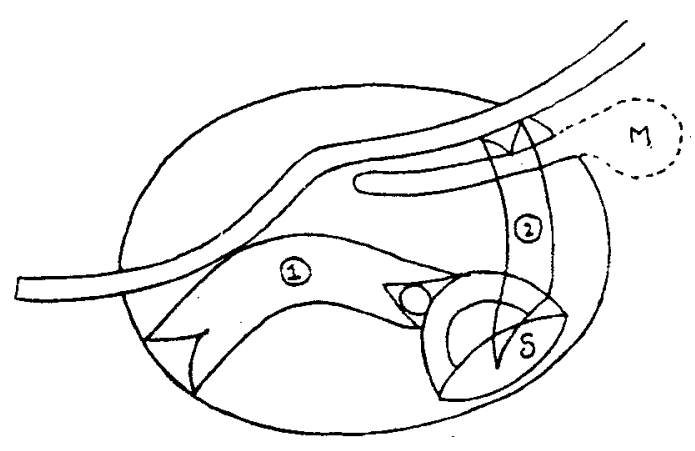

(1) Single Piece Cartilage

(2) Secord Support Cartilage

from malleus to Foot Plate
1. As single piece

2. As support to the malleus from owniche if malleus present or to the annulus if malleus absent.

Dr Kailash Sant
Pune

For replacement of incus, my preferred choice is a shaped and repositioned autograft. This is possible in most of the cases and I have no hesitation in the incus even in cholesteatomatous ear. I make sure to run the burr over the incus completely to denude the Incus of any epithelium before doing so. When the patients incus is completely destroyed, then I like using stored homograft incus or cartilage. In 1994,1 looked at a series of 100 cases of autograft incus repositioning 1 had done and followed up for over 3 years and noted a result of $82 \%$ with A$B$ gap to within $15 \mathrm{db}$.

\section{Dr Mohan Kameswaran Madras}

In descending order of preference, my choice for incus replacement in ossiculoplasty are autoincus ( if not too diseased), homoincus (preserved in absolute alchohol). Plester's gold prosthesis, teflon and cartilage ( conchal or tragal). The success rates encountered by me were :

Autoincus $70-80 \%$

Homoincus $70-80 \%$

Plester's gold Prosthesis : $50-60 \%$

( failures mainly due to displacement \& prosthesis)

Teflon $20-30 \%$

Cartilage $50-60 \%$

\section{Dr Gauri Mankekar Mumbai}

Regarding ossiculoplasty - for incus replacement

A. malleus present, stapes intact

Cartilage graft - Tragal cartilage conchal cartilage

Remnant of Incus by transposition tecnique

Thin bone - chip 
I have no experience with gold Prosthesis and TORP and PORP.

\section{Dr Dipali Solanky Surat}

In case of the handle of malleus and stapes are intact, I would prefer to use auto or homoincus sculpted to fit between the malleus handle and stapes head. However, in case incus is not available, Gold prosthesis ( partial ossicular replacement prosthesis) is my preference. If the prosthesis will be in contact with the tympanic membrane as in the case of absent malleus, then a thin slice of autograft tragal cartilage will be interposed between the prosthesis and the tympanic membrane reducing chances of extrusin providing stability to the prosthesis.

\section{Dr Samir Bhargava Mumbai}

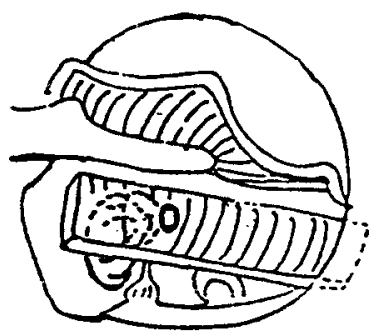

Fig: 1 Gold Porp placcd on the head of the stapes. The shaft of the prosthesis is inserted lage platfrom extending from the hypo tympanum to the epitympanum.

With increase in incidence of AIDS we prefer to use auto incus provided it is good enough to be refashioned. It is reshaped, socket is created to accommodate stapes head and groove is made for malleus handle. If malleus is retracted than tensor tympani may be cut. If malleus is necrosed and removed then we usually augment. Stapes head with conchal cartilage or reshaped bone. If auto incus is not available then preserved (70\% alcohol) homoincus is preferred over
PORP. Most PORPs extrude even if the cartilage slice is kept between PORP and tympanic membrane.

\section{Dr Hetal patel Mumbai}

For incus replacement, I routinely use the composite gold prosthesis mounted on an autograft cartilage platform, which extends from the epitympanum to just medical to the annulus in the hypotympanum. The main advantages are the simplicity of inserting the prosthesis, the stability, the prevention of retraction pockets and the cost-effectiveness. I find this the simplest and most reliable technique of incus replacement and the short term results ( 5 year follow up) are excellent. However, long term follow up is

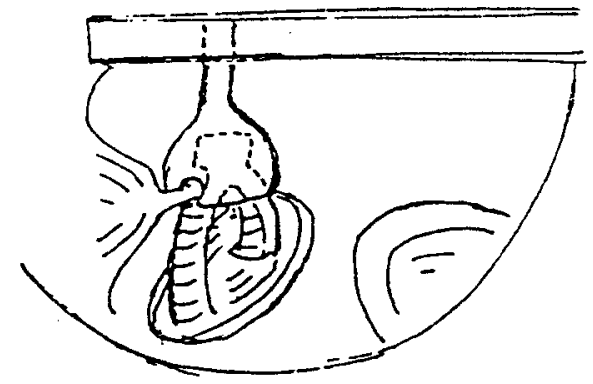

Fig: 2 Side view of the gold PORP sitting on the head of stapes. The stability of the $P$ achieved by the cartilage platform.

required to further validate our current results.

Dr Aruna Visvanathan

New Questions Coimbatore

Q. 1. How will you treat a child who has accidentally swallowed a corrosive solution?

Q. 2. What are the common manifestations of gastro Oesophageal Reflux in day to day clinical practice? 